\title{
Spontaneous Intraventricular Hemorrhage: When Should Intraventricular tPA Be Considered?
}

\author{
Peter A. Abdelmalik, MD, PhD ${ }^{1}$ Wendy C. Ziai, MD, MPH ${ }^{1}$ \\ ${ }^{1}$ Division of Neurosciences Critical Care, Departments of Neurology \\ and Anesthesia and Critical Care Medicine, The Johns Hopkins \\ University School of Medicine, Baltimore, Maryland \\ Address for correspondence Wendy C. Ziai, MD, MPH, Division of \\ Neurosciences Critical Care, Departments of Neurology, Anesthesia \\ and Critical Care Medicine, The Johns Hopkins University School of \\ Medicine, 600 N Wolfe St., Phipps 455, Baltimore, MD 21287 \\ Semin Respir Crit Care Med 2017;38:745-759. \\ (e-mail: weziai@jhmi.edu).
}

\begin{abstract}
Spontaneous intracerebral hemorrhage $(\mathrm{ICH})$ is the most common cause of intraventricular hemorrhage (IVH) in adults. Complicating approximately $40 \%$ of ICH cases, IVH adds to the morbidity and mortality of this often fatal form of stroke. It is also a severity factor that complicates subarachnoid hemorrhage and traumatic brain injury, along with other less common causes of intracranial bleeding. Medical and surgical interventions to date have focused on limiting $\mathrm{ICH}$ and $\mathrm{IVH}$ expansion, controlling intracranial pressure, and relieving obstructive hydrocephalus. The placement of an external ventricular drain (EVD) can achieve the latter two goals but has not demon-

\section{Keywords}

- intracerebral hemorrhage

- intraventricular hemorrhage

- fibrinolysis

- thrombolysis

- hydrocephalus

- Graeb

- alteplase

- external ventricular drain

- ventriculostomy strated improvement in clinical outcomes beyond mortality reduction. More recently, intraventricular fibrinolysis, utilizing the EVD, has gained interest as a safe and potentially effective method to maintain catheter patency and facilitate hematoma removal. A recent phase III clinical trial evaluating the efficacy of intraventricular alteplase versus intraventricular saline showed a mortality benefit, but failed to meet the primary endpoint of significant functional improvement. However, planned subgroup analysis focusing on patients with IVH volume $>20 \mathrm{~mL}$, and those with IVH removal $>85 \%$ suggest that significant functional benefits may be attainable with this therapy. The practice of intraventricular fibrinolysis for spontaneous IVH is not the standard of care; however, based on 20 years of experience, it meets thresholds as a safe intervention, and in those patients with a high burden of intraventricular blood, aggressive clearance may lead to improved quality of life in survivors of this morbid syndrome.
\end{abstract}

Rare are the syndromes in modern medicine that can cause sudden death so rapid that it goes unexplained even after an extensive postmortem examination. Acute obstructive hydrocephalus-caused by a tumor, cyst, or, more commonly, intraventricular hemorrhage (IVH)-leads to a rapid increase in intracranial pressure (ICP), accompanied by autonomic dysfunction and rostrocaudal herniation of the brainstem which can potentially lead to a very rapid sudden death. ${ }^{1}$
Prior to the routine use of computed tomography (CT) scans of the head, IVH was classically diagnosed at autopsy, with evidence of extravasated blood obstructing the ventricular system. Because of the nature of this finding, it was felt that IVH was universally fatal. However, with modern neuroimaging, IVH has been characterized extensively and ranges from mild layering of blood, typically in the posterior horn of the lateral ventricle, to complete casting of all ventricles. ${ }^{2}$
Issue Theme Advancements in Neurocritical Care and Emergency Neurology; Guest Editors: David Y. Hwang, MD, FNCS, and David M. Greer, MD, MA, FCCM, FAHA, FNCS, FAAN, FANA
Copyright (C) 2017 by Thieme Medical Publishers, Inc., 333 Seventh Avenue, New York, NY 10001, USA. Tel: +1(212) 584-4662.
DOI https://doi.org/ 10.1055/s-0037-1607991. ISSN 1069-3424. 
IVH can be classified into two categories: primary IVH and secondary IVH. Most cases of IVH are secondary, where an intracerebral hemorrhage (ICH) ruptures into the ventricle. ${ }^{3}$ Spontaneous ICH is the most common cause of IVHapproximately 40 to $45 \%$ have concomitant $\mathrm{IVH}^{4}$-followed by 13 to $28 \%$ of aneurysmal subarachnoid hemorrhage (SAH) presenting with IVH. $^{5}$ Other secondary etiologies include traumatic brain injury (TBI), arteriovenous malformation (AVM), venous sinus thrombosis, and moyamoya disease. Risk factors for IVH associated with spontaneous ICH include older age, higher baseline ICH volume, mean arterial pressure (MAP) above $120 \mathrm{~mm} \mathrm{Hg}$, and deep subcortical location of the primary hematoma in close proximity to ventricles. ${ }^{6}$ Primary IVH, defined as a nontraumatic intracranial hemorrhage confined to the ventricular system and immediate parenchymal ependymal lining, is a rare and less well-understood neurological disease, and is a diagnosis of exclusion. ${ }^{7}$ These lesions, with a reported incidence of 3 to $7 \%$, should be investigated with cerebral angiography to rule out structural pathology such as choroid plexus AVM and may have a higher risk of ventricular rehemorrhage with intraventricular fibrinolytic (IVF) therapy compared with secondary IVH. Dural AVMs can also rarely present with primary IVH presumably due to retrograde venous drainage creating backflow into subependymal veins causing their rupture. ${ }^{8}$ Any vascular or mass lesion defined on neuroimaging as the cause of IVH should be considered a contraindication to IVF with exception of a secured intracranial aneurysm. ${ }^{9}$

ICH is a deadly form of stroke, with 30-day mortality rates of 35 to $52 \%{ }^{4,10}$ The addition of IVH to ICH is a wellestablished independent predictor of increased mortality, with estimates ranging from 50 to $80 \%{ }^{4,6,10-12}$ In a seminal prospective study of 129 ICH patients, those who demonstrated IVH $(n=47)$ had a 30 -day mortality of $43 \%$, a rate independently related to IVH volume on multivariate analysis. $^{13}$ Similarly, Hallevi et al found that $45 \%$ of $406 \mathrm{ICH}$ patients demonstrated IVH, and were more than two times as likely to have death or disability compared with $\mathrm{ICH}$ patients without IVH. ${ }^{14}$ Functionally, IVH is associated with significantly worse scoring on indices of health-related quality of life, with specific deficits in mobility, self-care, and activities of daily living in survivors. ${ }^{15}$

Incidence rates of $\mathrm{ICH} / \mathrm{IVH}$ are increasing with the aging population and the increased prescription of anticoagulation and antithrombotic medications, for a variety of indications. ${ }^{10}$ Patients with anticoagulant-associated ICH more often demonstrate IVH extension than those without use of oral anticoagulation. ${ }^{16}$

The pathophysiology of spontaneous IVH involves vessel rupture into the ventricle from parenchymal $\mathrm{ICH}$ through ventricular wall erosion under the parenchymal hemorrhage, and distention of regional subependymal vessels with some vessels occluded by thrombus. ${ }^{17}$ Ependymal erosion may be the primary event or may occur secondary to the presence of intraventricular blood. Small parenchymal hemorrhagic infarcts at the ventricular edge which presumably rupture through the ependyma have also been described. ${ }^{18}$
Pathologically, IVH causes neurologic injury via three major mechanisms: ${ }^{19}$

1. Obstructive hydrocephalus.

2. Local damage to neighboring structures-including diencephalic structures such as the thalamus which are crucial for consciousness and cognition-by mechanisms of mechanical compression, infiltration of inflammatory mediators, and edema exacerbated by blood breakdown products.

3. The presence of blood breakdown products and inflammatory mediators within the cerebrospinal fluid (CSF) cisterns, causing inflammation and fibrosis at the level of CSF granulations, exacerbating nonobstructive hydrocephalus, and sometimes resulting in the need for long-term CSF diversion.

While typically presenting at the ictus of the injury, IVH may also be a delayed phenomenon. Maas and colleagues investigated the onset of delayed IVH ( $\mathrm{dIVH})$ in a prospective data cohort, reporting that $21 \%$ ( 23 of 112 patients) developed dIVH, $43 \%$ required a ventriculostomy, and this was an independent predictor of death and poor outcome. ${ }^{20}$ dIVH was associated with significantly greater ICH expansion and higher rates of craniotomy. Possible risk factors for dIVH included atrial fibrillation, higher rate of warfarin use and presenting INR, and hematoma location in the lentiform nucleus. Conversely, in another prospectively studied cohort, dIVH was noted in $14.5 \%$ (19 of 131 patients), but was not an independent predictor of death or disability once hematoma expansion was controlled for in multivariate analysis. ${ }^{21}$ In a pooled analysis of the 1,310 ICH patients recruited into the INTERACT clinical trials, 107 demonstrated dIVH, which again was an independent predictor of poor outcome in patients with small and moderate sized $\mathrm{ICH}^{22} \mathrm{dIVH}$ was associated with vitamin $\mathrm{K}$ antagonist use, ICH volume, ICH growth, and higher NIH stroke scale score on presentation. ${ }^{22}$ The significance of dIVH for intraventricular thrombolytic therapy is that it may represent patients with greater risk of IVH reexpansion or a need for a longer pretreatment stabilization period. In the first 300 patients enrolled in the landmark CLEAR III trial (the results of which are discussed extensively later in this review), time from diagnostic CT to last IVH expansion was a median of 5.6 hours with range of 1.5 to 50.5 hours indicating that hemorrhage stability may require an individualized approach with serial CT based on risk factor assessment when applying interventional treatments to $\mathrm{ICH}^{23}$

\section{Quantification of IVH: Bedside Scoring Tools with Clinical Utility}

The simple presence of blood within the ventricles can have prognostic consequences. It is included as a variable in the ICH score, which predicts 30-day mortality after $\mathrm{ICH}^{24}$ in the modified Fisher score which predicts vasospasm after $\mathrm{SAH},{ }^{25,26}$ and in TBI, where the presence of IVH on CT after TBI was associated with diffuse axonal injury as an independent predictor in a prospective study of 140 TBI patients. ${ }^{27}$

Although the volume of a parenchymal clot can be approximated to the volume of a cylinder using the $\mathrm{ABC} / 2$ 
paradigm, ${ }^{28}$ the configuration and anatomy of the ventricles do not allow for such an approximation. ${ }^{29}$ Nevertheless, several investigators have attempted to quantify the amount of blood within the ventricles, in the absence of computerized volumetric analyses, by assigning a score to each ventricular compartment on CT scan, and correlating the score to clinical outcomes.

The Graeb score, initially described by Graeb and colleagues in 1982, is a semiquantitative score which ranges from 0 to 12 . A maximum score of 4 is assigned to each of the right and left lateral ventricles, if there is blood filling the ventricle and is accompanied by ventricular expansion. The third and fourth ventricles are ascribed a maximum score of 2 each for being filled and expanded. ${ }^{30}$ Morgan and colleagues (2013) suggested, however, that the original Graeb score lacked the ability to differentiate specific regions of the ventricular system, and thus was unable to recapitulate true IVH volume. ${ }^{31}$ They instead formulated the modified Graeb score (mGS), where scores are assigned to specific ventricular compartments, that is, a maximum score of 4 is ascribed to each of the right, left, third, and fourth ventricles, in addition to a maximum score of 2 to each right and left temporal horn and each right and left occipital horn (-Fig. 1). An additional point of 1 is given to each compartment if it is expanded beyond its normal anatomic limits due to hematoma, for a maximum score of 32 . Both the Graeb and mGS scores have been extensively validated in outcome studies. $^{4,29-32}$ The mGS has been validated in ICH patients from both clinical trials and a consecutive hospital-based study. From the CLEAR IVH trial (phase II) and the Virtual International Stroke Trials Archive (VISTA) with more than 400 participants, mGS was predictive of poor outcome (modified Rankin scale [mRS] $>3$ ), whereas the original Graeb score was not. ${ }^{31}$ The strength of the mGS to predict outcome in IVH patients was also assessed in the Lund stroke registry, where $198 \mathrm{ICH}$ patients were evaluated, 86 of who had IVH. ${ }^{32}$ Each point increase in mGS increased the odds for a poor 90 -day functional outcome ( $\mathrm{mRS}>3$ ) by $11 \%$, comparing favorably with a $12 \%$ increase in the odds of a poor outcome for each point increase from aforementioned clinical trial data. The mGS therefore applies as an independent predictor of death or poor functional outcome in both research and nonselected IVH populations. ${ }^{32}$

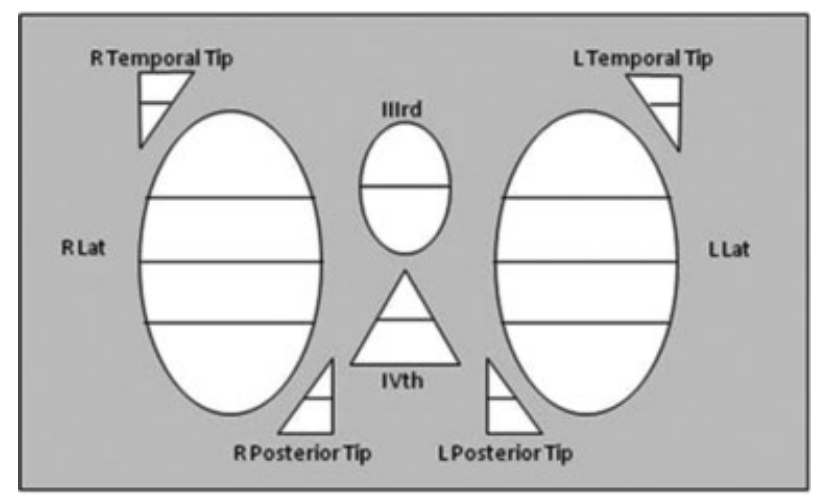

Fig. 1 The modified Graeb scale. ${ }^{31}$
Another score, proposed in 2009 by Hallevi and colleagues, ${ }^{29}$ termed the IVH score, is calculated as follows: each lateral ventricle scores 0 (no blood or small amount of layering), 1 (up to one-third filled with blood), 2 (one-third to twothirds filled with blood), or 3 (mostly or completely filled with blood)-for a total of 6 maximal points. The third and fourth ventricles score 0 for no blood or 1 if partially or completely filled with blood. Hydrocephalus is coded as present (1) or absent (0), for a potential total score of $9 .{ }^{29}$ This score demonstrated excellent correlation with software-derived IVH volumes in a retrospective analysis of 175 cases. Furthermore, total volume, consisting of IVH score and ICH volume using the $\mathrm{ABC} / 2$ estimation, was able to predict mortality and poor outcome with greater sensitivity and specificity than either the original Graeb score or ICH score alone. ${ }^{29}$

Therefore, although software-based volumetric analysis of IVH has become the standard in clinical trials, simple bedside estimation of IVH severity has proven useful for early prognostication and evaluation of clot resolution with therapeutic removal.

\section{Medical and Surgical Management of Spontaneous IVH Prior to Thrombolytic Therapy}

The presence of IVH is one of the several indicators that a patient requires intensive care unit (ICU) level care, based on the Triage ICH model. ${ }^{33}$ The presence of IVH was a predictor of ICU admission and for ICU stay longer than 5 days in the INTERACT2 trial for intensive blood pressure (BP) management in $\mathrm{ICH}^{34}$ Additionally, there are secondary complications that have been associated with IVH, such as an increased rate of nosocomial infection. ${ }^{35}$

Our current care for IVH is predominantly driven by management guidelines for spontaneous ICH as published by the American Heart Association (AHA) ${ }^{36}$ and European Stroke guidelines ${ }^{37}$ and involves brain monitoring and ICU support in the setting of gradually accumulating evidence for primary clot $(\mathrm{ICH})$ reduction.

Blood pressure management has been the mainstay of therapy for acute spontaneous ICH, with the goal of limiting hematoma expansion. The CLEAR III trial required BP stability according to AHA guidelines in the 6-hour period prior to start of intraventricular study agent. Current guidelines for BP control for ICH patients recommends (Class 1/Level of Evidence A) lowering systolic BP to $140 \mathrm{~mm} \mathrm{Hg}$, provided there are no contraindications in patients with a presenting systolic $\mathrm{BP}$ of 150 to $220 \mathrm{~mm} \mathrm{Hg}{ }^{36}$ It is important to recognize that this degree of BP reduction may be potentially harmful in a patient with IVH causing obstructive hydrocephalus and elevated ICP. Most (75\%) patients in the INTERACT2 trial presented with mild to moderate size $(<20 \mathrm{~mL})$ hematomas and therefore maintaining adequate cerebral perfusion pressure (CPP) was less of a concern. Moreover, just as INTERACT2 showed no significant effect of intensive BP-lowering treatment on hematoma growth, it is not clear whether acute lowering of systolic BP has an effect on minimizing IVH growth. Pooled analysis of INTERACT1 and INTERACT2, where ICH 
patients were randomized to goal systolic BP of $<140$ or $<180 \mathrm{~mm} \mathrm{Hg}$, demonstrated no clear effect of aggressive BP control on IVH growth between groups. ${ }^{38}$ Adjusted mean IVH growth was nonsignificantly greater in patients with a mean achieved systolic BP $\geq 160 \mathrm{~mm}$ Hg over 24 hours. Risk factors for IVH growth $(>2 \mathrm{~mL})$ in the first 24 hours in the FAST trial (recombinant activated factor VII [rFVIIa]) did, however, include baseline MAP greater than $120 \mathrm{~mm} \mathrm{Hg}$, along with larger baseline ICH volume. ${ }^{6}$ From these studies, it is reasonable to maintain a systolic $\mathrm{BP}<160 \mathrm{~mm} \mathrm{Hg}$ during thrombolytic treatment, provided that CPP (MAP minus ICP) is preserved. AHA guidelines currently recommend a CPP of 50 to $70 \mathrm{~mm} \mathrm{Hg}$ in patients undergoing ICP monitoring, depending on the status of cerebral autoregulation (Class IIb; Level of Evidence C). ${ }^{36}$ Analysis of CPP data from the CLEAR III trial suggests that maintaining MAP at the top end of this range may be associated with improved survival (Ziai W, MD, MPH, unpublished data, February 2017).

Prior to consideration of intraventricular thrombolysis, the decision for extraventricular drain (EVD) placement is made. Most often performed at the bedside, the EVD can relieve elevated ICP, evacuate intraventricular blood and blood breakdown products that may cause chemical meningitis, ${ }^{39}$ and is associated with decreased short-term mortality. $^{40}$ EVD placement has never been subjected to a randomized controlled trial (RCT) for spontaneous IVH and remains controversial regarding indications for use and outcomes benefit, likely due to the multifactorial mechanisms of brain injury. ${ }^{41}$ AHA guidelines suggest that the use of an EVD is reasonable, especially in patients with decreased level of consciousness (Class IIa/Level of Evidence $\mathrm{B}^{36}$ ).

While EVDs are common practice, the threshold (both clinically and radiographically) for EVD insertion after IVH varies considerably. Generally, the presence of hydrocephalus (radiographically) and a deteriorating neurologic examination are indications for placing an EVD. From a retrospective analysis of 563 patients with spontaneous ICH and $25.2 \%$ of EVD use, the odds of EVD use increased with younger age, and with increasing IVH volume, along with lower ICH volume and ICH located outside of brainstem. ${ }^{42}$ EVD use, which included use of intraventricular thrombolytics in more than one-third (36.2\%) of cases, was associated with a trend toward lower mortality, but simultaneously was also associated with higher (worse) mRSs. ${ }^{42}$ Mortality benefit at 30 days from EVD use was significantly associated with higher ICH severity indicators including patients with ICH score of 4 , greater ICH volume, and lower initial GCS in propensity score-adjusted analyses. Similarly, in a retrospective review of 183 patients with spontaneous IVH, EVD placement occurred in $37 \%$ of patients-with independent predictors of EVD placement being GCS $<8$, Graeb score $>5$, and nonlobar ICH $>30 \mathrm{~mL}$. In this study, with relatively high EVD use and exclusion of intraventricular thrombolytic cases, placement was an independent predictor of reduced mortality for patients with GCS $>3 .{ }^{43}$ Another retrospective analysis with an EVD usage rate of $28.6 \%$ reported early EVD use in over $80 \%$ within $6.4 \pm 3.3$ hours of presentation, with delayed insertion after
48 hours in a minority of patients. Independent associations with EVD insertion were similar: GCS score $<8$, total mGS $>13$, and-in particular-fourth ventricle $\mathrm{mGS}=5(>75 \%$ filled with blood + dilatation) ${ }^{44}$ The CLEAR IVH and other randomized trials of intraventricular thrombolytics ( - Table 1) have all utilized pragmatically placed EVDs.

\section{Brief History of Intraventricular Thrombolytics}

The rationale for thrombolytics for evacuation of IVH originated from the observation that EVD placement does not immediately clear the IVH and is often complicated by obstruction from blood clots and debris, which reduce patency and slow or stop blood removal. EVDs are therefore most often placed on the side opposite the greatest volume of ventricular hematoma to avoid obstruction, although this review will promote the concept that more rapid clearance of IVH occurs when an EVD is placed in the lateral ventricle with the largest clot volume. ${ }^{45}$ The standard EVD catheter has an inner diameter of $1.5 \mathrm{~mm}$ to limit parenchymal injury, although large bore catheters with inner diameter of $2.3 \mathrm{~mm}$ are typically used in cases with IVH. In a prospective database regarding EVD insertion and practice for all indications, $19 \%$ of 98 patients required at least one EVD replacement, and $41 \%$ developed at least one temporary occlusion. ${ }^{46}$ In a retrospective analysis of 227 patients with IVH requiring EVD, large bore catheters decreased the risk of catheter obstruction without any increase in catheter-related hemorrhages, and a nonsignificant decrease in catheter-related infections was also noted. Despite this, mortality was higher in the large bore catheter group, likely due to a selection bias where patients with larger volume hematomas were selected to receive the large bore catheters. ${ }^{47}$

Over the past 20 years, there has been significant interest in the use of IVF to facilitate blood removal and maintain EVD patency. Experimental studies of thrombolytic-mediated clot removal have consistently demonstrated improved blood clot removal, hydrocephalus, and inflammation. ${ }^{45,46,48-51}$ In 2000, a systematic review of 16 retrospective studies $(n=201)$ with severe IVH (Graeb score $\geq 7$ ) secondary to $\mathrm{ICH}$ reported that combined therapy with EVD placement and IVF was associated with a significantly lower mortality rate $(9 \%)$ and lower risk for unfavorable outcome (22\%), as compared with patients treated with EVD alone (mortality 56\%; poor outcome: $87 \%) .{ }^{40}$ In 2002 , a Cochrane database systematic review of 10 independent studies ( 8 case series/ retrospective studies, 1 quasi-RCT, 1 RCT) using IVF found anecdotal evidence supporting safety and possible therapeutic value. ${ }^{52}$ Nearly a decade later, a systematic review of four randomized and eight observational studies comparing EVD alone to EVD combined with IVF in severe IVH due to spontaneous supratentorial ICH reported overall mortality risk decreased from 46.7\% (EVD alone) to 22.7\% (EVD + IVF). ${ }^{53}$ IVF was also associated with an increase in good functional outcome and with no significant difference between the two groups in terms of shunt dependence or complications, although the rebleeding rate was greater in 


\begin{tabular}{|c|c|c|c|c|}
\hline 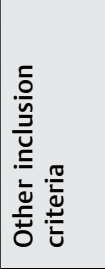 & 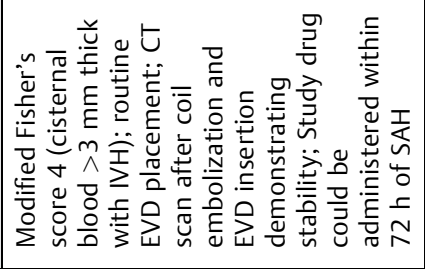 & 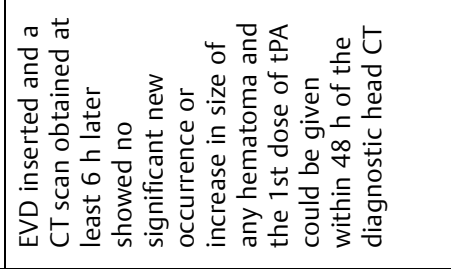 & 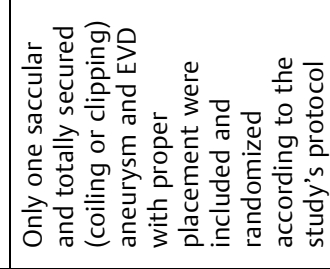 & 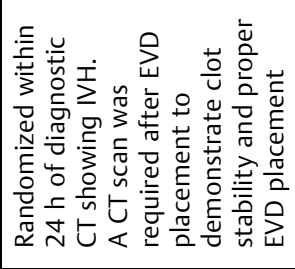 \\
\hline 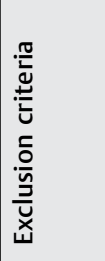 & 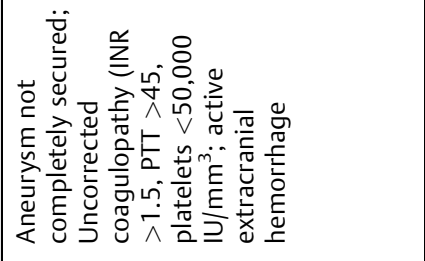 & 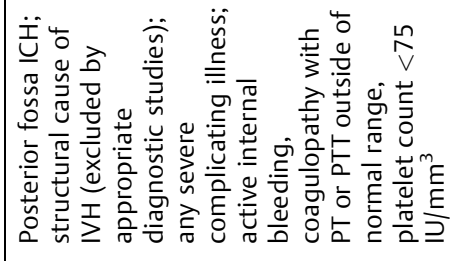 & 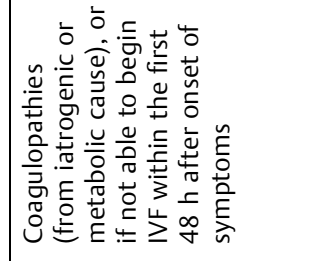 & 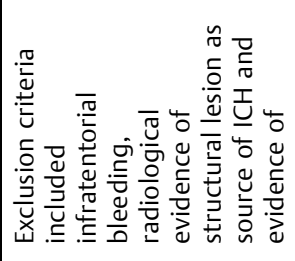 \\
\hline 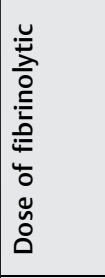 & 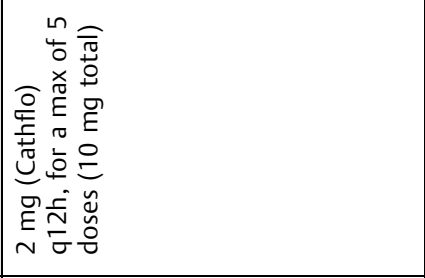 & 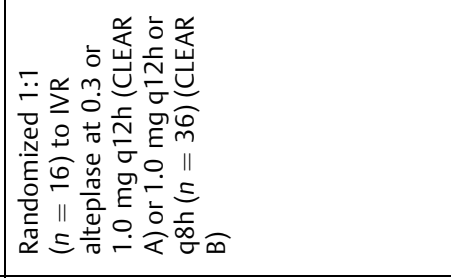 & 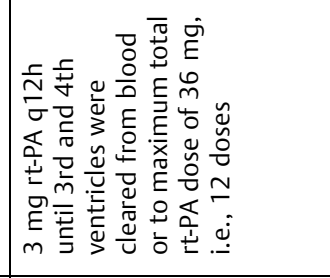 & 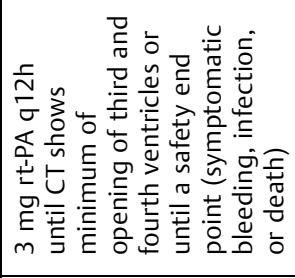 \\
\hline 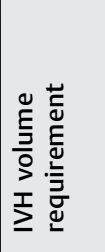 & 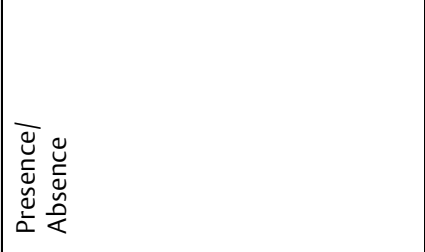 & 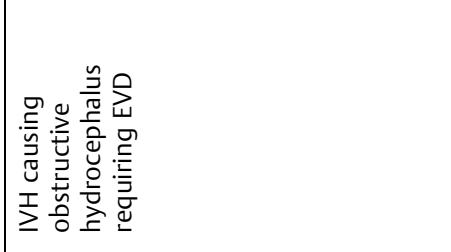 & 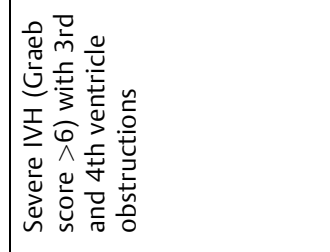 & 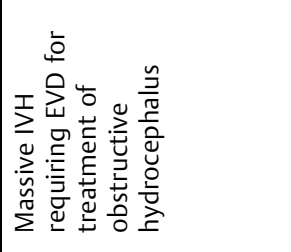 \\
\hline 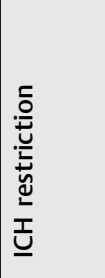 & $\frac{\pi}{z}$ & 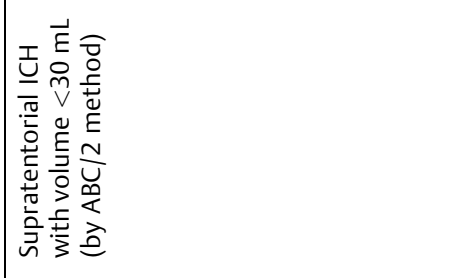 & $\frac{s}{z}$ & 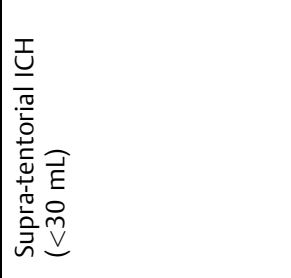 \\
\hline $\begin{array}{l}\bar{\lambda} \\
\text { 严 }\end{array}$ & $\stackrel{\infty}{\wedge}$ & $\stackrel{\infty}{\wedge}$ & 足 & $\left|\begin{array}{l}n \\
0 \\
\infty \\
\underline{\infty}\end{array}\right|$ \\
\hline 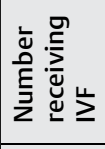 & 0 & $\simeq$ & $=$ & $\stackrel{\sim}{\sim}$ \\
\hline 竞 & $\simeq$ & $\stackrel{d}{\sim}$ & 9 & $\stackrel{\infty}{+}$ \\
\hline 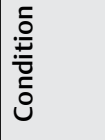 & 売 & $\underline{I}$ & 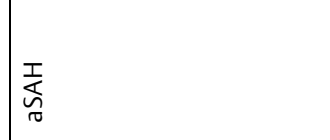 & $\underline{I}$ \\
\hline 窟离 & 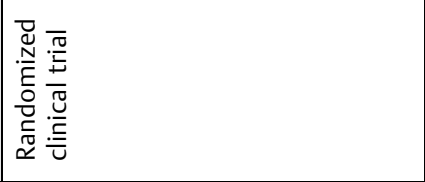 & 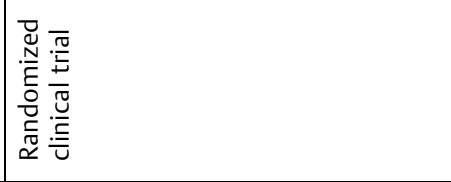 & 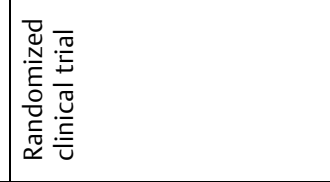 & 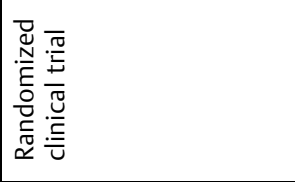 \\
\hline 줄 & 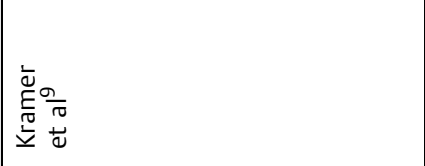 & 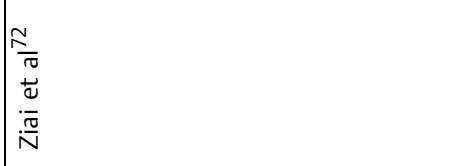 & 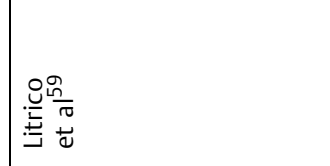 & 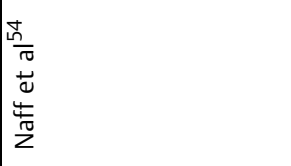 \\
\hline
\end{tabular}




\begin{tabular}{|c|c|c|c|c|c|c|c|}
\hline 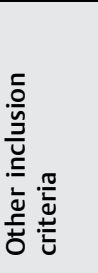 & & 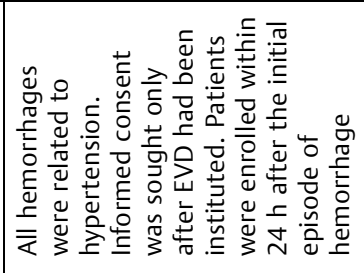 & 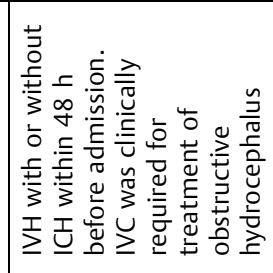 & 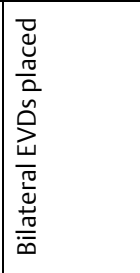 & 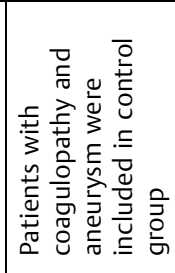 & 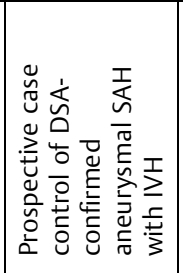 & 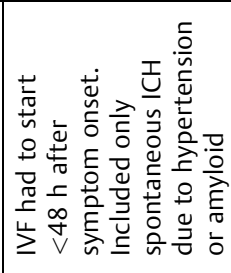 \\
\hline 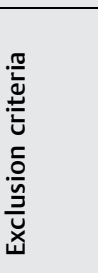 & 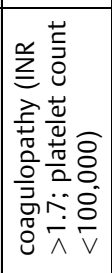 & 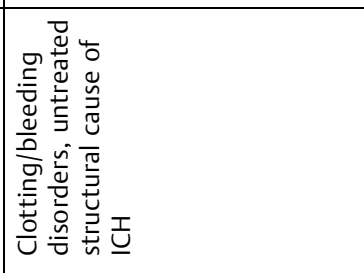 & 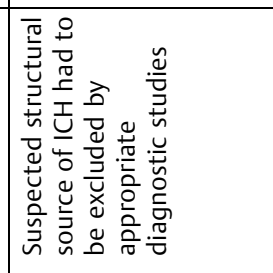 & 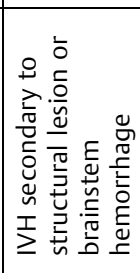 & $\mid \frac{1}{2}$ & $\frac{\pi}{z}$ & $\frac{\pi}{z}$ \\
\hline 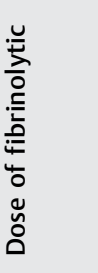 & & 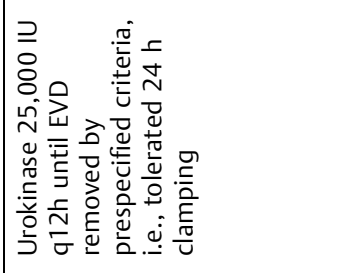 & 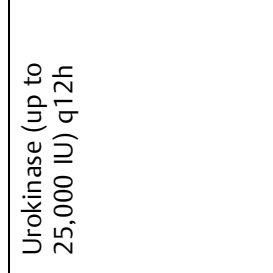 & 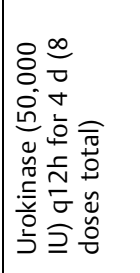 & 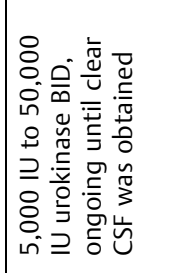 & 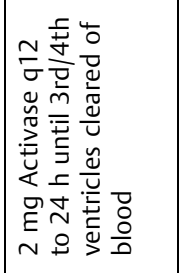 & 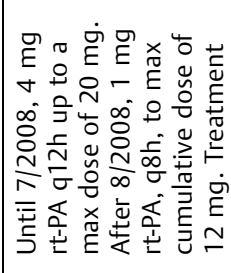 \\
\hline 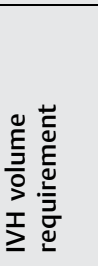 & & 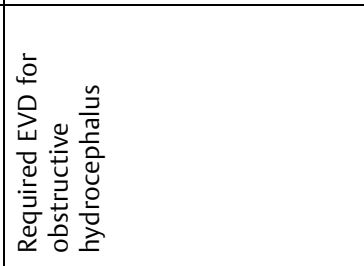 & 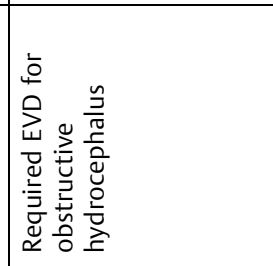 & 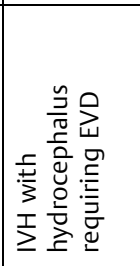 & 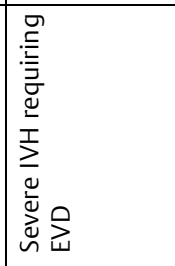 & 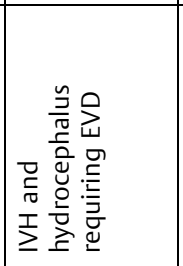 & 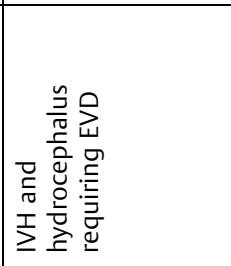 \\
\hline 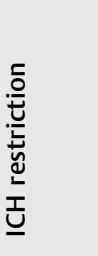 & & 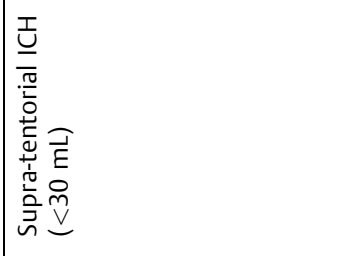 & 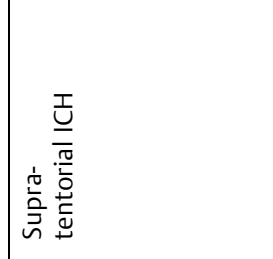 & 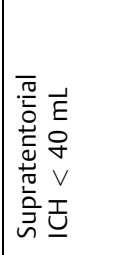 & $\frac{\pi}{z}$ & $\frac{\alpha}{z}$ & 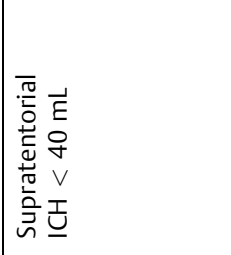 \\
\hline 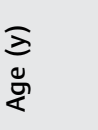 & & $\stackrel{\infty}{\wedge}$ & $\stackrel{\infty}{\wedge}$ & 织 & m & $\begin{array}{l}\widehat{O} \\
\stackrel{N}{N} \\
\text { Ñ }\end{array}$ & 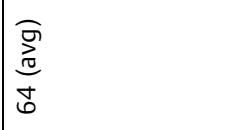 \\
\hline 吝 & & $r$ & $\nabla$ & $\stackrel{\circ}{\circ}$ & $\wedge$ & $\stackrel{\circ}{-}$ & ठ \\
\hline 莺 & & $\simeq$ & $\infty$ & $\bar{\sim}$ & $\stackrel{\circ}{\circ}$ & & $\stackrel{\infty}{\sim}$ \\
\hline 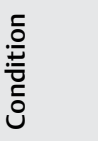 & & $\underline{\underline{I}}$ & $\underline{I}$ & $\underline{I}$ & $\underline{I}$ & 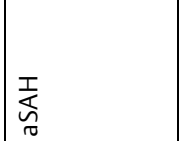 & $\underline{I}$ \\
\hline 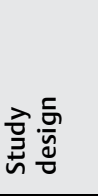 & & 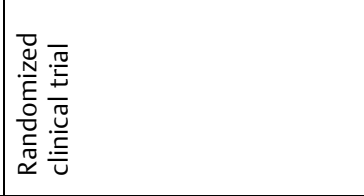 & 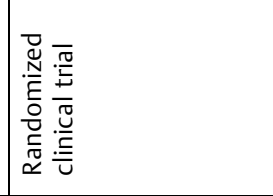 & 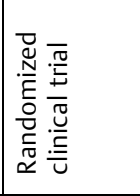 & 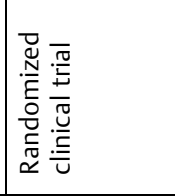 & 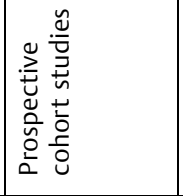 & 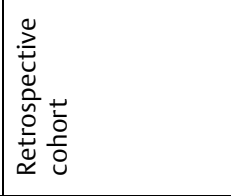 \\
\hline 离 & & 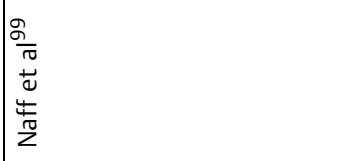 & $\mid \begin{array}{l}\frac{8}{0} \\
\frac{0}{\pi} \\
\frac{\pi}{4} \\
\frac{4}{\pi} \\
z\end{array}$ & $\frac{\bar{\sigma}}{\sigma \frac{0}{\pi}}$ & 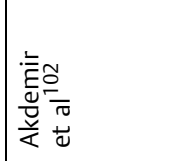 & 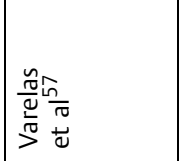 & 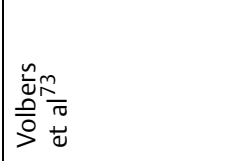 \\
\hline
\end{tabular}




\begin{tabular}{|c|c|c|c|c|c|c|}
\hline 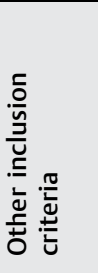 & 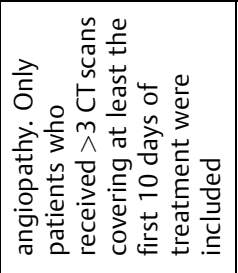 & 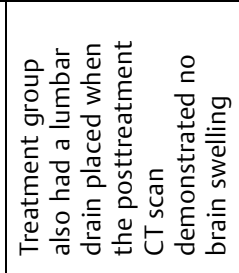 & 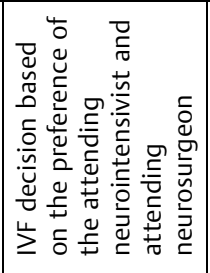 & 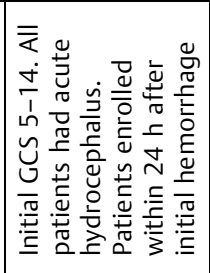 & 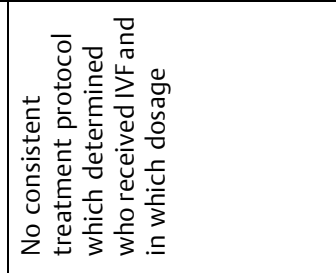 & 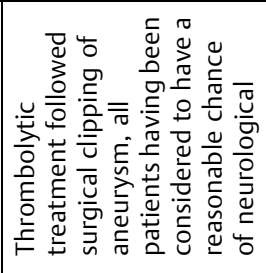 \\
\hline 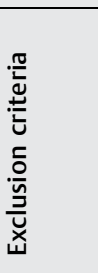 & & 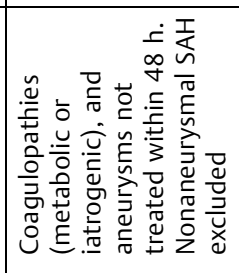 & 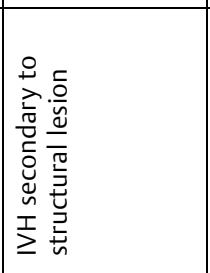 & 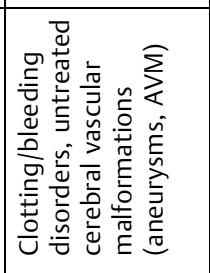 & 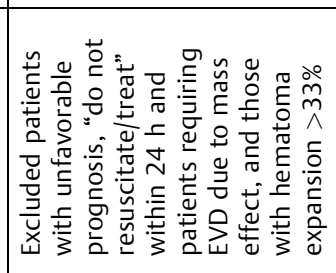 & $\frac{\pi}{2}$ \\
\hline 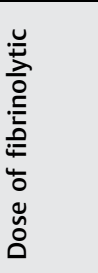 & 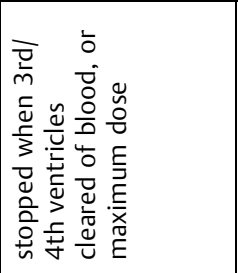 & 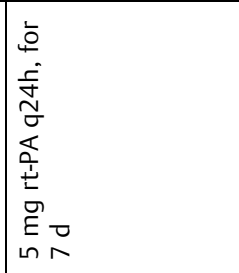 & 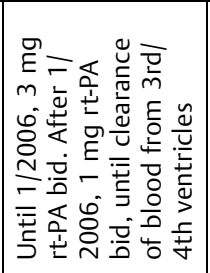 & 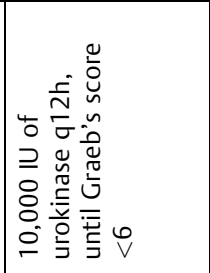 & 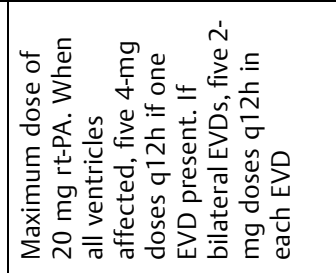 & 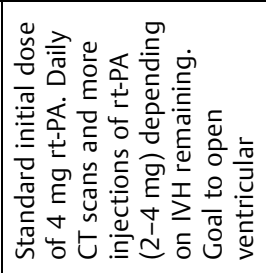 \\
\hline 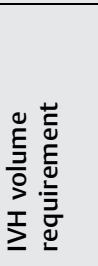 & & 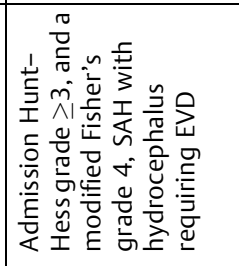 & 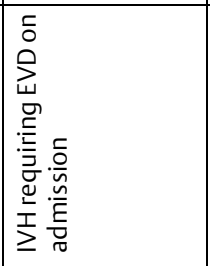 & 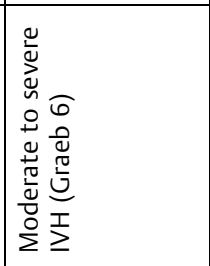 & 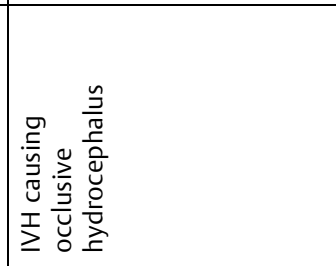 & 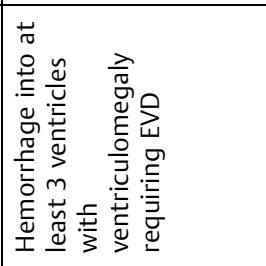 \\
\hline 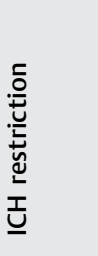 & & $\frac{\pi}{z}$ & 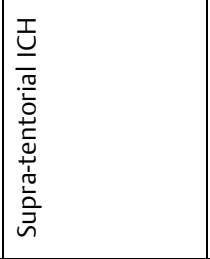 & $\begin{array}{l}\vec{E} \\
\dot{D} \\
\underline{V} \\
\underline{I} \\
\underline{U}\end{array}$ & 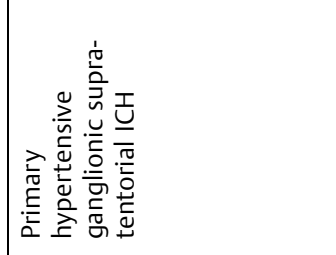 & $\frac{\kappa}{z}$ \\
\hline $\begin{array}{l}\widehat{ } \\
\overline{0} \\
\text { gू }\end{array}$ & & 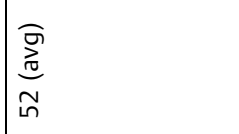 & 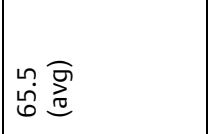 & $\begin{array}{l}0 \\
1 \\
\infty \\
1 \\
\end{array}$ & 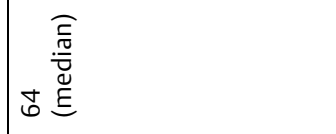 & 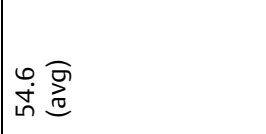 \\
\hline 兑 & & $\bar{\sigma}$ & $\stackrel{m}{\sim}$ & $\stackrel{\Xi}{\square}$ & $\approx$ & $\bar{N}$ \\
\hline 营 & & $\stackrel{2}{\circ}$ & $\stackrel{\circ}{m}$ & $\stackrel{\infty}{\sim}$ & $\dot{F}$ & $\stackrel{\circ}{m}$ \\
\hline 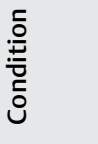 & & 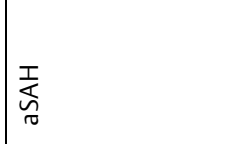 & $\underline{I}$ & $\underline{I}$ & $\underline{I}$ & 焉 \\
\hline 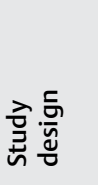 & & 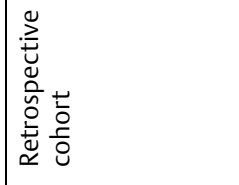 & 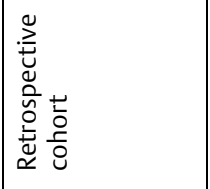 & 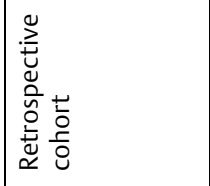 & 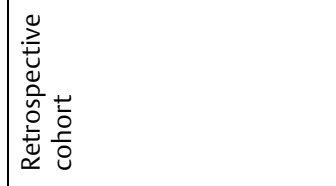 & 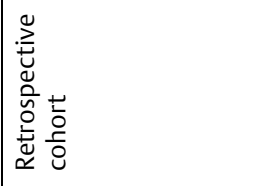 \\
\hline 忞 & & 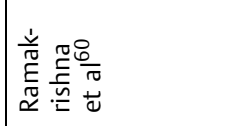 & 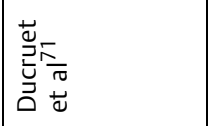 & 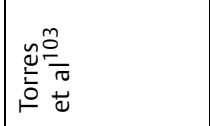 & 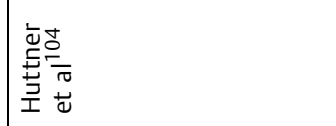 & 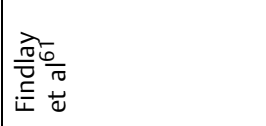 \\
\hline
\end{tabular}




\begin{tabular}{|c|c|c|c|c|}
\hline 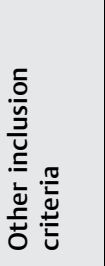 & 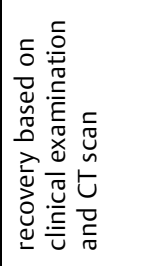 & 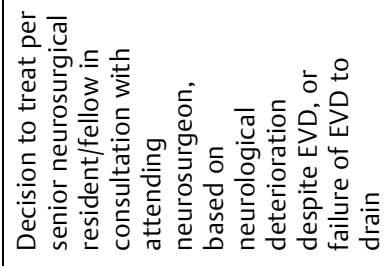 & 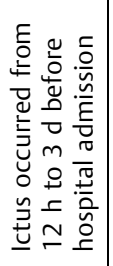 & $\frac{\mathbb{s}}{2}$ \\
\hline 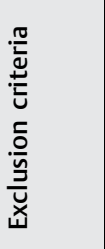 & & 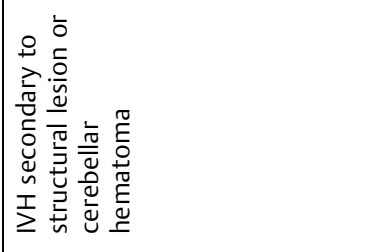 & $\frac{\mathbb{s}}{z}$ & $\frac{\Delta}{z}$ \\
\hline 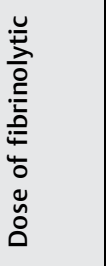 & 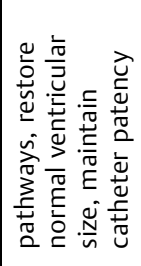 & 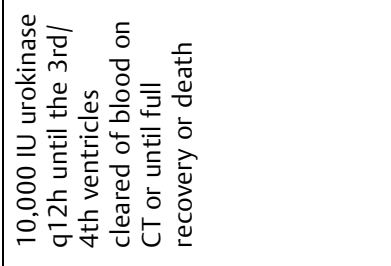 & 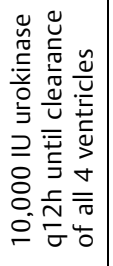 & 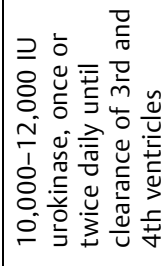 \\
\hline 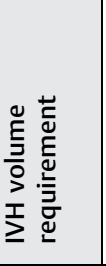 & & 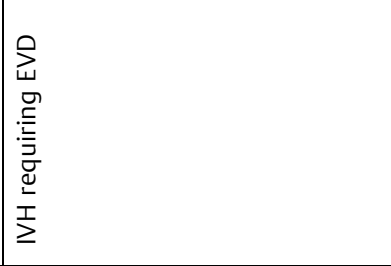 & 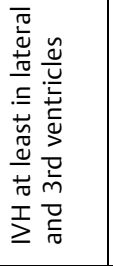 & 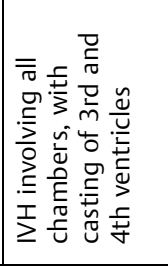 \\
\hline 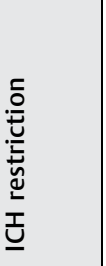 & & 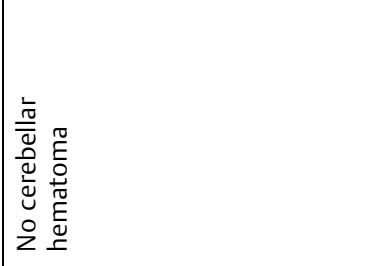 & 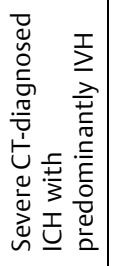 & \\
\hline 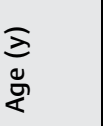 & & \begin{tabular}{l} 
ñ \\
$\hat{1}$ \\
\multirow{2}{\sim}{}
\end{tabular} & 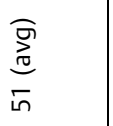 & 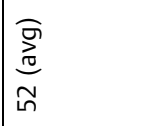 \\
\hline 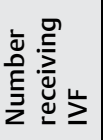 & & $\approx$ & $\stackrel{\bullet}{\circ}$ & 0 \\
\hline 莺 & & 우 & $\bar{v}$ & $=$ \\
\hline 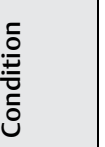 & & $\underline{I}$ & $\underline{I}$ & 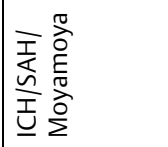 \\
\hline 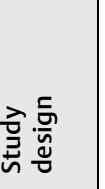 & & 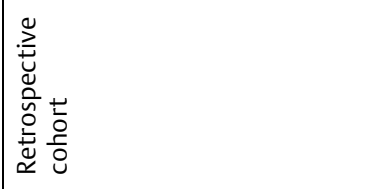 & 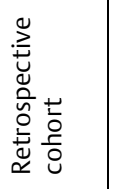 & 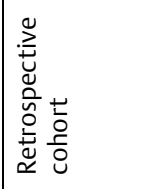 \\
\hline 总 & & 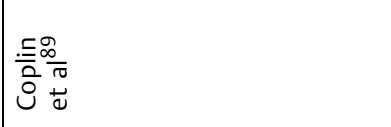 & 峁 & 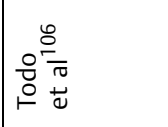 \\
\hline
\end{tabular}


the IVF group (11.4 vs. $6.4 \%$ in the EVD alone group). The mortality benefit was highly significant with urokinase, but not with recombinant tissue-type plasminogen activator (tPA), a finding raising the question of whether therapeutic effect of thrombolysis may differ by choice of agent. That same year, the Phase I Intraventricular Hemorrhage Treatment Trial was published by Naff and colleagues, who randomized 48 patients to receive intraventricular alteplase or saline and reported an excellent safety profile while demonstrating some benefit in decreasing mortality. ${ }^{54}$

Staykov et al also performed a systematic review of 23 studies (1993-2010; $n=436) .{ }^{19}$ The mortality rate was $53 \%$ $(n=133)$ for EVD alone and $16 \%(n=212)$ for EVD plus thrombolysis, compared with $71 \%(n=91)$ in conservatively treated patients. Unfavorable outcome was higher in the IVF group compared with other systematic reviews (45 vs. $70 \%$ for EVD alone vs. $86 \%$ for conservative treatment) but again suggested significant potential relative benefit from IVF.

Finally, an updated systematic review including eight RCTs demonstrated consistent results of mortality reduction with IVF by nearly half (compared with EVD alone), increased odds of good functional outcome by $66 \%$, and decreased rate of shunt dependence (relative risk [RR], 0.62). ${ }^{55}$ Ventriculitis and rehemorrhage rates were not increased with IVF.

These reviews all promote IVF as an effective strategy to reduce mortality and improve functional outcome, without significant increase in complication rates. Unfortunately, most data in these reviews came from small randomized and nonrandomized prospective/retrospective studies with considerable heterogeneity among patient populations, dosing regimens, length of follow-up, and etiologies (including both ICH and SAH in some). The findings of these metaanalyses do, however, support real-world data. In a retrospective review using the National Inpatient sample, 34,044 patients with spontaneous IVH were identified, $3.3 \%$ of who received intraventricular alteplase. These patients demonstrated a significant decrease in in-hospital mortality and a trend toward favorable discharge (i.e., home or inpatient rehabilitation) compared with EVD alone, without a concomitant increase in meningitis, nor any difference in chronic shunt diversion procedures. ${ }^{56}$

Intraventricular alteplase has proven to be a safe intervention, not only in patients with IVH after ICH, but also in patients with IVH due to $\mathrm{SAH} .{ }^{9,57}$ In a randomized placebocontrolled trial of intraventricular alteplase versus saline in 12 patients with modified Fisher scale $4 \mathrm{SAH}$ who had undergone aneurysm embolization and EVD placement, no new ICH developed. This small proof of concept trial demonstrated a significant reduction in intraventricular blood without any difference in neurological outcome at 6 months (a secondary outcome). ${ }^{9}$

\section{Patient Selection for Intraventricular Thrombolysis}

To understand the selection of IVH subjects for intraventricular thrombolysis, it is worthwhile to consider the focused patient selection in CLEAR III and in prior research studies
(-Table 1). The CLEAR IVH trials restricted entry to patients younger than 80 years with spontaneous (hypertensive) ICH volume $<30 \mathrm{~mL}$; obstruction of the third and/or fourth ventricles presenting within 24 hours of symptom onset; and stability of ICH, IVH, and any EVD tract hemorrhage prior to 72 hours from diagnostic CT scan. ${ }^{54,58}$ Patients in other studies prior to CLEAR III all generally excluded IVH associated with pregnancy, significant coagulopathy, intracranial tumor, AVM, cerebellar/brainstem hematoma, or intracranial aneurysm (-Table 1). Several small studies of IVF in IVH associated with aneurysmal SAH have shown promise for this therapy in patients with secured aneurysms. 9,57,59-61

Results from the CLEAR III trial itself cannot be generalized to patients with large ICH $(>30 \mathrm{~mL}$ ) or unstable hemorrhages, baseline mRS $>1$, age $>80$ years, Glasgow Coma Scale of 3, or probable amyloid angiopathy. Reversal of warfarin-induced coagulopathy or thrombocytopenia was permitted in CLEAR III, although patients on novel anticoagulants (NOACs) were excluded.

Other potential treatment indications and issues either beyond the scope of the current evidence or undergoing further evaluation involve earliest time to safely start IVF, minimum and maximum IVH volume responding to therapy, how much blood needs to be removed, and application of concomitant therapeutic approaches along with IVF. With regard to time from ictus to time of IVF treatment, a CLEAR III subgroup analysis suggested a trend toward improved benefit from IVF in patients treated within 48 hours of ictus. ${ }^{58}$

\section{Lessons from the CLEAR III Trial Results}

The CLEAR III trial itself was a randomized, double-blinded, placebo-controlled, multicenter trial powered to establish the effectiveness of intraventricular alteplase for the treatment of IVH on functional outcome at 180 days, dichotomized as mRS 0 to 3 versus 4 to $6 .^{58}$ Power to observe was predicated on $40 \%$ mortality and baseline poor outcome of $78 \%$. Retention was excellent, with 6-month follow-up data available for $97.4 \%$ of patients. However, there was no statistically significant difference in good versus poor functional status at 180 days between the saline- and alteplasetreated groups: saline $45 \%$ versus alteplase $48 \%$, a difference of $3.5 \%(p=0.42)$ after adjustment for IVH volume and thalamic ICH location. While the primary outcome was neutral, there was a significant decrease in the odds of being dead at 180 days for the experimental group (alteplase 18\% vs. saline $29 \%$; hazard ratio: 0.60 ; $95 \%$ confidence interval [CI]: $0.41-0.86 ; p=0.006)$, which came at the expense of a significantly greater proportion of patients with mRS 5 (i.e., bedbound) compared with the saline group (17 vs. 9\%; $p=0.007$ ). There was, however, no difference in proportion of vegetative patients measured by extended Glasgow Outcome Scale (3\% in both groups), nor any difference in survivors in long-term facilities (alteplase $14 \%$ vs. saline $12 \% ; p=0.48)$.

Consistent with the previous trials, intraventricular alteplase was deemed to be safe, with a significantly lower rate of bacterial ventriculitis or serious adverse events when 
compared with intraventricular saline. The rate of symptomatic bleeding was the same in both groups (2\%). There was also a significant decrease in neurological, respiratory, and sudden deaths in the alteplase group, supporting the claim that intraventricular alteplase may correct life-threatening obstruction within the ventricular system.

In a planned post hoc analysis, patients with IVH volumes greater than $20 \mathrm{~mL}$ demonstrated a significant treatment effect of intraventricular alteplase, with a statistically significant increase in patients with good functional outcome (mRS: $0-3$ ) at 180 days (odds ratio: $1.87,95 \% \mathrm{CI}: 1.02$, $3.42) .{ }^{58}$ Furthermore, in those patients who achieved $>85 \%$ reduction in IVH volume, the primary outcome was significant for the alteplase-treated group after adjusting for confounders. $^{58}$ - Fig. 2 shows the planned secondary analysis relating 180-day mRS score to the amount of clot removal at the end of treatment in all participants, which shows that for any starting IVH volume, probability of good outcome increased with greater IVH volume reduction. Unfortunately, only $33 \%$ of the alteplase group reached the treatment endpoint of $80 \%$ IVH clot removal, a fact emphasizing the goal of successful treatment of obstructive hydrocephalus, manifested by radiographic clearance or "reopening" of the third and fourth ventricles on CT. This has often been used as the criterion for discontinuation of dosing in studies of IVF and was one of three stopping criteria in the CLEAR-IVH protocol. $^{19,62}$ The other two criteria, $80 \%$ removal of IVH clot volume and removal of mass effect, were less well emphasized and are major considerations for a follow-up trial to CLEAR III.

While it is unlikely that CLEAR III will immediately propel the practice of delivering intraventricular alteplase to standard practice in the treatment of IVH, its post hoc analyses suggest that with optimal patient selection, IVF may be quite efficacious. That is, in patients with hypertensive IVH greater than $20 \mathrm{~mL}$, early and aggressive clot removal, with a target of greater than $80 \%$ IVH clot reduction (rather than simply alleviating radiographic third and fourth ventricular obstruction) is a safe, life-saving practice that may contribute to enhancing the quality of life in survivors of this moribund disease. It is also worthwhile noting that failure of the CLEAR III trial to meet its primary outcome may be partially

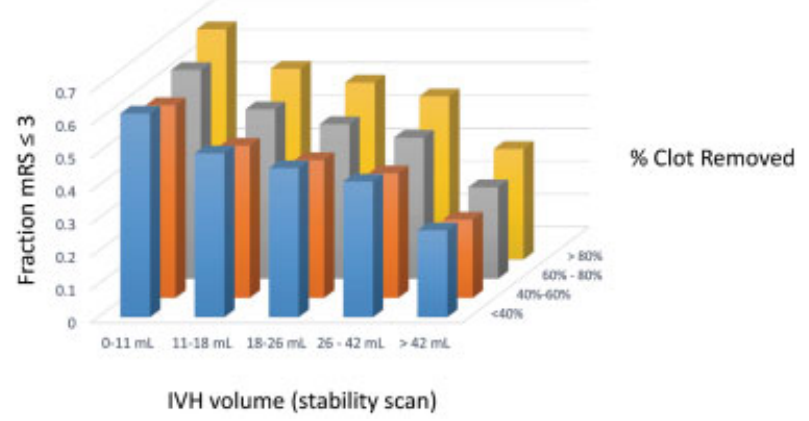

Fig. 2 Relationship between mRS 0-3 (good outcome) at 180 days and percent clot removed by starting intraventricular hemorrhage (IVH) volume as determined at stability prior to randomization. explained by better-than-expected outcomes in the control group which had their EVD flushed with saline every 8 hours, as compared with historical controls, for which flushing the ventriculostomy at regular intervals does not generally occur in routine practice. The proportion of saline-treated patients achieving a good functional outcome (mRS: 0-3) in CLEAR III was $45 \%$, while the pretrial power analysis, based on previous data, estimated good functional outcome in only $22 \%$ of the control group, ${ }^{63}$ thus leaving the trial with too few highseverity patients enrolled, and inadequately powered, to resolve this narrow difference between alteplase- and saline-treated groups.

\section{Enhancing Clot Lysis and Removal}

Several strategies to enhance IVH clot lysis and removal exist. One such strategy, in conjunction with IVF, is the placement of a second EVD in the contralateral lateral ventricle where typically the dominant IVH resides. Prior to the CLEAR III trial, two studies had evaluated the effect of bilateral EVDs on IVH clearance with IVF, with different conclusions. ${ }^{64,65}$ Staykov et al evaluated 27 patients with spontaneous basal ganglionic ICH $<40 \mathrm{~mL}$ and obstructive IVH, comparing 13 patients treated through a single and 14 through bilateral EVDs with IVF. ${ }^{64}$ No difference was found in IVH clot resolution between the two groups-either as total IVH volume reduction or reduction within segmented lateral, third, or fourth ventricles. A trend toward higher incidence of ventriculitis in the bilateral EVD group was reported. In this study, initial median IVH volume was between 30 and $35 \mathrm{~mL}$ in each group. ${ }^{64}$ Conversely, in 14 patients from the CLEAR IVH trials with IVH $>40 \mathrm{~mL}$, bilateral EVD use versus single $\operatorname{EVD}$ ( $n=7$ in each group) led to increased clot resolution, in the presence of both alteplase and saline, ${ }^{65}$ suggesting that multiple catheters may be an effective, safe strategy to achieve the $80 \%$ benchmark for the removal of IVH under conditions of large IVH ( $>40 \mathrm{~mL}$ ) with casting and mass effect. These data are also supported by a small randomized study of 47 patients with severe IVH (Graeb score $\geq 5$ ) who were randomized to placement of one or two EVDs and reported significantly faster drainage of IVH volume with two EVDs compared with one, together with IVF and no incidence of rehemorrhage or infection. ${ }^{66}$

A second strategy to improve clot removal efficiency is EVD placement position. In a post hoc analysis of data from the CLEAR III trial, clinically meaningful 85\% IVH volume reduction was independently associated with smaller IVH volume, younger age, and placement of ventricular drainage catheters ipsilateral to the dominant IVH volume, adjusting for alteplase treatment. ${ }^{67}$ It has therefore been proposed that while initial EVDs indicated for ICP control may be targeted away from the intraventricular clot to maximize CSF drainage, placement of a second EVD directly into the ventricular clot may be necessary to effectively reduce IVH volume with fibrinolytic.

Fibrinolysis after intraventricular alteplase demonstrates first-order kinetics, which is saturable. ${ }^{68}$ A higher dose may, in theory, increase IVH clearance. The dose of 
intraventricular alteplase used in the CLEAR III trial was $1 \mathrm{mg}$ (in $1 \mathrm{~mL}$ of saline), delivered every 8 hours. This was the product of dose-finding trials, where clot resolution rates were not dose dependent across doses of $0.3,1$, and $3 \mathrm{mg}$ when looking at the total IVH clearance (all ventricles together). Clearance of the third and fourth ventricle, however, did demonstrate a dose-dependent relationship. ${ }^{69}$ In the Phase I CLEAR IVH trial, a dose of $3 \mathrm{mg}$ every 12 hours was also associated with a nonsignificantly higher symptomatic rehemorrhage rate which has not been observed with lower doses. ${ }^{69}$ Experimentally, higher doses of intraventricular alteplase have led to increased intraventricular leukocytosis and edema of periventricular tissues and choroid plexus in both hemispheres in rat models of $\mathrm{IVH}^{70}$ In a clinical report of increased perihematomal edema with intraventricular alteplase, Ducruet and colleagues used doses of $3 \mathrm{mg}$ of alteplase, ${ }^{71}$ while others, who did not see significant increases in perihematomal edema, used a $1 \mathrm{mg}$ dose. $^{72,73}$

At this time, individualized dosing for IVF has not been studied. Variations in response to IVF with alteplase is a subject of future research and has been identified in related cohorts with radiographic features ${ }^{74}$ and clot-related factors. ${ }^{75}$ Additionally, genetic factors modulating the response to intravenous alteplase for revascularization after acute ischemic stroke have been characterized, including Apo E2 phenyotype, ${ }^{76}$ polymorphisms at loci of the factor XIII gene, ${ }^{77}$ thrombin-activatable fibrinolysis inhibitor gene, ${ }^{78}$ interleukin 1B gene, and von Willebrand factor genes. ${ }^{79}$ Furthermore, the risk for hemorrhagic transformation with higher serum levels of matrix metalloproteinase-9, involved in the integrity of the blood-brain barrier, has been reported. $^{80}$

Another proposed method for more aggressive IVH clearance is the use of an EVD with fibrinolysis in combination with lumbar drainage. Together, it is postulated that clearance of blood from the ventricles and removal of inflammatory mediators may exacerbate hydrocephalus and CSF flow and absorption would be enhanced. This has been tested in an open label randomized trial of 30 patients which was stopped early after interim analysis demonstrated $43 \%$ of the control group requiring permanent CSF diversion compared with no patients undergoing combination therapy. ${ }^{81}$ There was no difference in functional outcome at 90 days (a secondary outcome), nor was there a significant difference in IVH clearance between control and experimental groups, as noted by the change in Graeb score (from 9 to 2) over 10 days in each group. ${ }^{81}$ The results of the recently completed EARLYdrain trial assessing outcomes after early lumbar CSF drainage in aneurysmal SAH may influence this practice in spontaneous IVH patients. ${ }^{82}$

\section{Hydrocephalus and Intracranial Hypertension as Indications for Intraventricular Fibrinolysis}

Hydrocephalus occurs in 20 to $40 \%$ of all IVH patients, ${ }^{83-87}$ although again, the clinical benefit of EVD is debated. ${ }^{84}$
Obstructive hydrocephalus on admission CT is an independent predictor of increased in-hospital and 30-day mortality $^{83,85}$ and also of 6-month neurologic outcome for caudate ICH. ${ }^{86}$ In the STICH I trial (Surgical Treatment for ICH) that studied outcomes after surgical evacuation of ICH, favorable outcomes were less frequent when IVH was present and significantly lower when hydrocephalus was also present. ${ }^{87}$ In two prospective observational cohorts of patients presenting with spontaneous ICH, those patients demonstrating IVH had a significantly elevated odds ratio of having gait dysfunction and incontinence at 3 months. ${ }^{88}$

These studies suggest that CSF diversion may not only be life-saving but also beneficial for long-term quality of life in survivors. However, it should be noted that in an older prospective database of $81 \mathrm{ICH}$ patients, the 40 patients with hydrocephalus had a significantly higher mortality and poor functional outcome, which did not change if an EVD was placed. ${ }^{83}$ Also, while an EVD may alleviate hydrocephalus by diverting CSF, a retrospective analysis of EVD use in patients with ICH with IVH suggested that hydrocephalus did not improve and ventriculomegaly continued despite CSF drainage, with no benefit on mortality. ${ }^{84}$

Interestingly, ICP is not always elevated in spontaneous IVH despite obvious obstructive hydrocephalus. In a retrospective analysis of $40 \mathrm{IVH}$ patients who received an EVD, the mean initial ICP reported was $15.6 \mathrm{~mm} \mathrm{Hg}$, and 15\% had ICP elevation $>20 \mathrm{~mm} \mathrm{Hg}$ at the time of EVD insertion. ${ }^{89}$ In a prospective evaluation of $11 \mathrm{IVH}$ patients demonstrating obstructive IVH with serial ICP measurement every 4 hours, $14 \%$ of ICP readings were greater than $20 \mathrm{~mm} \mathrm{Hg} .{ }^{90}$ However, an analysis of an IVH cohort from a large prospective randomized study of thrombolysis for IVH in 100 patients, also with serial ICP recordings, ${ }^{91}$ demonstrated that continuous drainage of CSF contributes to the normalization of ICP and that the proportion of ICP readings $>30 \mathrm{~mm} \mathrm{Hg}$ per patient was an independent predictor of morbidity (mRS: 46 ) and mortality at 30 days. In this analysis, threshold events $>20$ and $>30 \mathrm{~mm} \mathrm{Hg}$ were more frequent in placebo versus alteplase-treated groups after adjustment for confounders, suggesting that fibrinolytic therapy may have a beneficial effect on ICP control in this condition. While ICP is not frequently elevated in patients presenting with severe IVH, ICP values $>30 \mathrm{~mm} \mathrm{Hg}$ do correlate with short-term mortality. 91

An additional, and important, therapeutic intervention provided by the insertion of an EVD is to allow for removal of blood and blood breakdown products. Blood and blood degradation products can remain embedded within the arachnoid granulations, and impede CSF flow, and with prolonged exposure, permanently occlude and scar the arachnoid granulations and consequently inhibit CSF absorption causing nonobstructive (communicating) hydrocephalus. $^{92}$ Furthermore, ventricular dilatation with blood affects patient outcomes in a manner independent of the effects related to clot volume or mass effect, a fact supporting the hypothesis that there is an independent biochemical effect of ventricular blood on outcomes. ${ }^{93}$ A hypothesis based on animal data suggests that ICH with IVH causes more 
hydrocephalus than IVH alone due to iron overload, and that both anatomical and histological changes are reversible with deferoxamine, an iron chealator. ${ }^{94}$

\section{Rebleeding and Intraventricular Fibrinolysis}

The most feared complication of IVF is rebleeding. As a result, protocols demand demonstration of hematoma stability, correction of any coagulopathy, and exclusion of any vascular anomaly as a cause for bleeding. From the structured screening process used in the CLEAR III trial, of 10,538 consecutive IVH cases prospectively screened, 496 cases (4.7\%) screened negative for underlying vascular lesion, met the inclusion criteria, and were enrolled in the trial; 1,205 cases $(11.4 \%)$ were concurrently screened and excluded from the trial because of a demonstrated underlying vascular lesion. ${ }^{95}$ It is therefore recommended that all patients under consideration for IVF treatment undergo vascular neuroimaging to rule out underlying lesions which may increase hemorrhage risk. The low risk of symptomatic rebleeding in the CLEAR III trial ( $2 \%$ in each treatment groups) likely reflects a stringent stabilization practice which required all sources of bleeding (ICH, IVH, catheter tract) to be stable for 12 hours before starting IVF. In their review, Staykov et al found that rebleeding associated with IVF occurred in approximately $5 \%$ of cases

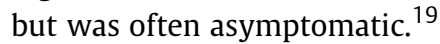

As a result, the current 2015 AHA guidelines for the management of ICH patients suggest IVF appears safe, while acknowledging the efficacy and safety of this treatment strategy remain uncertain (Class IIb/Level of Evidence $\mathrm{B}^{36}$ ).

\section{Beyond Intraventricular Fibrinolysis}

In addition to EVD-based catheter evacuation of IVH, there has been considerable interest in surgical and microsurgical techniques for the removal of blood from the ventricles. The 2015 AHA guidelines state that the efficacy of endoscopic treatment of IVH is uncertain (Class IIb/Level of Evidence B) ${ }^{36}$ however, this may be changed in updated guidelines, as minimally invasive surgery has come to the forefront as a viable option for treating IVH. For example, Chen and colleagues have reported on their experience using minimally invasive endoscopic surgery for thalamic hemorrhages with IVH and hydrocephalus in 48 patients which demonstrated a shorter length of stay and reduced need for ventriculoperitoneal (VP) shunting. However, there was no significant difference in mortality or functional outcomes at 30 and 90 days. ${ }^{96}$ Prior to this study, a trial in 2007 randomized 44 patients with IVH to management with EVD with IVF using urokinase or to endoscopic evacuation, with IVF utilized if the IVH volume was greater than $10 \mathrm{~mL}$. The authors reported no statistical difference in mortality but a significant improvement in functional outcome at 2 months in the neuroendoscopy group. ${ }^{97}$ A meta-analysis comparing neuroendoscopic surgery combined with EVD for IVH versus the combination of EVD and IVF, which included 680 patients, noted significant benefits in mortality, good functional outcome, hematoma evacuation rate, and VP shunt depen- dence, ${ }^{98}$ suggesting that the use of endoscopic techniques with EVD may be a feasible option in the future.

\section{Conclusions}

IVH contributes significantly to the morbidity and mortality of ICH. The pathophysiological mechanisms include acute obstructive hydrocephalus, which can lead to sudden death, in addition to increased ICP and inflammation. The standard of care for the management of IVH causing obstructive hydrocephalus remains placement of an EVD for CSF diversion and ICP control. The insertion of an EVD may be lifesaving in the acute setting to relieve obstructive hydrocephalus; however, ICP crisis is not common among IVH patients, and routine EVD placement for CSF drainage is not sufficient for the clearance of intraventricular blood in a manner that is clinically beneficial.

Intraventricular thrombolysis with alteplase, at a dose of $1 \mathrm{mg}$ every 8 hours, is safe, and may have beneficial effects on outcome in patients with a significant burden of intraventricular blood (i.e., $>20 \mathrm{~mL}$ ), if treatment is successful in removing $>80 \%$ of IVH. Such treatment, in turn, is postulated to decrease inflammation and subsequent fibrosis within the ependymal and subarachnoid membranes. Other methods of efficient blood removal from the ventricles include use of lumbar drainage and microsurgical techniques, which have shown promise in the evacuation of large collections of intraventricular blood and may gain favor as more data become available.

\section{Disclosures}

- Peter Abdelmalik: No relevant financial disclosures.

- Wendy Ziai: Supported by grants 5U01NS062851 and 1U01NS08082.

\section{References}

1 Black M, Graham DI. Sudden unexplained death in adults caused by intracranial pathology. J Clin Pathol 2002;55(01):44-50

2 Dey M, Stadnik A, Awad IA. Thrombolytic evacuation of intracerebral and intraventricular hemorrhage. Curr Cardiol Rep 2012; 14(06):754-760

3 Ojemann RG, Heros RC. Spontaneous brain hemorrhage. Stroke 1983;14(04):468-475

4 Hanley DF. Intraventricular hemorrhage: severity factor and treatment target in spontaneous intracerebral hemorrhage. Stroke 2009;40(04):1533-1538

5 Mohr G, Ferguson G, Khan M, et al. Intraventricular hemorrhage from ruptured aneurysm. Retrospective analysis of 91 cases. J Neurosurg 1983;58(04):482-487

6 Steiner T, Diringer MN, Schneider D, et al. Dynamics of intraventricular hemorrhage in patients with spontaneous intracerebral hemorrhage: risk factors, clinical impact, and effect of hemostatic therapy with recombinant activated factor VII. Neurosurgery 2006;59(04):767-773, discussion 773-774

7 Sanders E. A study of primary, immediate, or direct hemorrhage into the ventricles of the brain. Am J Med Sci 1881;82:85-128

8 Irie F, Fujimoto S, Uda K, et al. Primary intraventricular hemorrhage from dural arteriovenous fistula. J Neurol Sci 2003; 215(1-2):115-118

9 Kramer AH, Roberts DJ, Holodinsky J, et al. Intraventricular tissue plasminogen activator in subarachnoid hemorrhage patients: a 
prospective, randomized, placebo-controlled pilot trial. Neurocrit Care 2014;21(02):275-284

10 Qureshi AI, Mendelow AD, Hanley DF. Intracerebral haemorrhage. Lancet 2009;373(9675):1632-1644

11 Young WB, Lee KP, Pessin MS, Kwan ES, Rand WM, Caplan LR. Prognostic significance of ventricular blood in supratentorial hemorrhage: a volumetric study. Neurology 1990;40(04): 616-619

12 Tuhrim S, Dambrosia JM, Price TR, et al. Intracerebral hemorrhage: external validation and extension of a model for prediction of 30-day survival. Ann Neurol 1991;29(06):658-663

13 Tuhrim S, Horowitz DR, Sacher M, Godbold JH. Volume of ventricular blood is an important determinant of outcome in supratentorial intracerebral hemorrhage. Crit Care Med 1999;27 (03):617-621

14 Hallevi H, Albright KC, Aronowski J, et al. Intraventricular hemorrhage: anatomic relationships and clinical implications. Neurology 2008;70(11):848-852

15 Delcourt C, Zheng D, Chen X, et al. Associations with healthrelated quality of life after intracerebral haemorrhage: pooled analysis of INTERACT studies. J Neurol Neurosurg Psychiatry 2017;88(01):70-75

16 Horstmann S, Rizos T, Lauseker M, et al. Intracerebral hemorrhage during anticoagulation with vitamin $\mathrm{K}$ antagonists: a consecutive observational study. J Neurol 2013;260(08): 2046-2051

17 Gordon A. Primary ventricular hemorrhage: further contribution to a characteristic symptom group. Arch Neurol Psychiatry 1938; 39:1272-1276

18 Gates PC, Barnett HJ, Vinters HV, Simonsen RL, Siu K. Primary intraventricular hemorrhage in adults. Stroke 1986;17(05): 872-877

19 Staykov D, Bardutzky J, Huttner HB, Schwab S. Intraventricular fibrinolysis for intracerebral hemorrhage with severe ventricular involvement. Neurocrit Care 2011;15(01):194-209

20 Maas MB, Nemeth AJ, Rosenberg NF, Kosteva AR, Prabhakaran S, Naidech AM. Delayed intraventricular hemorrhage is common and worsens outcomes in intracerebral hemorrhage. Neurology 2013;80(14):1295-1299

21 Witsch J, Bruce E, Meyers E, et al. Intraventricular hemorrhage expansion in patients with spontaneous intracerebral hemorrhage. Neurology 2015;84(10):989-994

22 Moullaali TJ, Sato S, Wang X, et al. Prognostic significance of delayed intraventricular haemorrhage in the INTERACT studies. J Neurol Neurosurg Psychiatry 2017;88(01):19-24

23 Ziai W, Ullman N, Thompson C, et al. Stabilizing Bleeding Prior To Acute Therapies for Spontaneous Intracerebral Hemorrhage. In: Abstract. 3215. International Stroke Conference (ISC), San Diego, CA; 2014

24 Hemphill JC III, Bonovich DC, Besmertis L, Manley GT, Johnston SC. The ICH score: a simple, reliable grading scale for intracerebral hemorrhage. Stroke 2001;32(04):891-897

25 Frontera JA, Claassen J, Schmidt JM, et al. Prediction of symptomatic vasospasm after subarachnoid hemorrhage: the modified fisher scale. Neurosurgery 2006;59(01):21-27, discussion 21-27

26 Claassen J, Bernardini GL, Kreiter K, et al. Effect of cisternal and ventricular blood on risk of delayed cerebral ischemia after subarachnoid hemorrhage: the Fisher scale revisited. Stroke 2001;32(09):2012-2020

27 Mata-Mbemba D, Mugikura S, Nakagawa A, et al. Intraventricular hemorrhage on initial computed tomography as marker of diffuse axonal injury after traumatic brain injury. J Neurotrauma 2015;32(05):359-365

28 Kothari RU, Brott T, Broderick JP, et al. The ABCs of measuring intracerebral hemorrhage volumes. Stroke 1996;27(08): 1304-1305
29 Hallevi H, Dar NS, Barreto AD, et al. The IVH score: a novel tool for estimating intraventricular hemorrhage volume: clinical and research implications. Crit Care Med 2009;37(03):969-974, e1

30 Graeb DA, Robertson WD, Lapointe JS, Nugent RA, Harrison PB. Computed tomographic diagnosis of intraventricular hemorrhage. Etiology and prognosis. Radiology 1982;143(01):91-96

31 Morgan TC, Dawson J, Spengler D, et al; CLEAR and VISTA Investigators. The Modified Graeb Score: an enhanced tool for intraventricular hemorrhage measurement and prediction of functional outcome. Stroke 2013;44(03):635-641

32 Hansen BM, Morgan TC, Betz JF, et al. Intraventricular extension of supratentorial intracerebral hemorrhage: the modified Graeb scale improves outcome prediction in Lund Stroke Register. Neuroepidemiology 2016;46(01):43-50

33 Klaas JP, Braksick S, Mandrekar J, et al. Factors associated with the need for intensive care unit admission following supratentorial intracerebral hemorrhage: the Triage ICH Model. Neurocrit Care 2017;27(01):75-81

34 Wartenberg KE, Wang X, Muñoz-Venturelli P, et al. Intensive care unit admission for patients in the INTERACT2 ICH blood pressure treatment trial: characteristics, predictors, and outcomes. Neurocrit Care 2017;26(03):371-378

35 Vial F, Brunser A, Lavados P, Illanes S. Intraventricular bleeding and hematoma size as predictors of infection development in intracerebral hemorrhage: a prospective cohort study. J Stroke Cerebrovasc Dis 2016;25(11):2708-2711

36 Hemphill JC III, Greenberg SM, Anderson CS, et al; American Heart Association Stroke Council; Council on Cardiovascular and Stroke Nursing; Council on Clinical Cardiology. Guidelines for the management of spontaneous intracerebral hemorrhage: a guideline for healthcare professionals from the American Heart Association/American Stroke Association. Stroke 2015;46(07): 2032-2060

37 Steiner T, Al-Shahi Salman R, Beer R, et al; European Stroke Organisation. European Stroke Organisation (ESO) guidelines for the management of spontaneous intracerebral hemorrhage. Int J Stroke 2014;9(07):840-855

38 Chan E, Anderson CS, Wang X, et al; INTERACT Investigators. Early blood pressure lowering does not reduce growth of intraventricular hemorrhage following acute intracerebral hemorrhage: results of the INTERACT studies. Cerebrovasc Dis Extra 2016;6(03):71-75

39 Hallevi H, Walker KC, Kasam M, Bornstein N, Grotta JC, Savitz SI. Inflammatory response to intraventricular hemorrhage: time course, magnitude and effect of t-PA. J Neurol Sci 2012;315(12):93-95

40 Nieuwkamp DJ, de Gans K, Rinkel GJ, Algra A. Treatment and outcome of severe intraventricular extension in patients with subarachnoid or intracerebral hemorrhage: a systematic review of the literature. J Neurol 2000;247(02):117-121

41 Dey M, Jaffe J, Stadnik A, Awad IA. External ventricular drainage for intraventricular hemorrhage. Curr Neurol Neurosci Rep 2012;12(01):24-33

42 Lovasik BP, McCracken DJ, McCracken CE, et al. The effect of external ventricular drain use in intracerebral hemorrhage. World Neurosurg 2016;94:309-318

43 Herrick DB, Ullman N, Nekoovaght-Tak S, et al. Determinants of external ventricular drain placement and associated outcomes in patients with spontaneous intraventricular hemorrhage. Neurocrit Care 2014;21(03):426-434

44 Hughes JD, Puffer R, Rabinstein AA. Risk factors for hydrocephalus requiring external ventricular drainage in patients with intraventricular hemorrhage. J Neurosurg 2015;123(06): 1439-1446

45 Jaffe J, Melnychuk E, Muschelli J, et al. Ventricular catheter location and the clearance of intraventricular hemorrhage. Neurosurgery 2012;70(05):1258-1263, discussion 1263-1264 
46 Fargen KM, Hoh BL, Neal D, O'connor T, Rivera-Zengotita M, Murad GJA. The burden and risk factors of ventriculostomy occlusion in a high-volume cerebrovascular practice: results of an ongoing prospective database. J Neurosurg 2016;124(06): 1805-1812

47 Gilard V, Djoubairou BO, Lepetit A, et al. Small versus large catheters for ventriculostomy in the management of intraventricular hemorrhage. World Neurosurg 2017;97:117-122

48 Liu DZ, Waldau B, Ander BP, et al. Inhibition of Src family kinases improves cognitive function after intraventricular hemorrhage or intraventricular thrombin. J Cereb Blood Flow Metab 2017;37 (07):2359-2367

49 Wagner KR, Xi G, Hua Y, et al. Ultra-early clot aspiration after lysis with tissue plasminogen activator in a porcine model of intracerebral hemorrhage: edema reduction and blood-brain barrier protection. J Neurosurg 1999;90(03):491-498

50 Mayfrank L, Kim Y, Kissler J, et al. Morphological changes following experimental intraventricular haemorrhage and intraventricular fibrinolytic treatment with recombinant tissue plasminogen activator. Acta Neuropathol 2000;100(05):561-567

51 Narayan RK, Narayan TM, Katz DA, Kornblith PL, Murano G. Lysis of intracranial hematomas with urokinase in a rabbit model. J Neurosurg 1985;62(04):580-586

52 Lapointe M, Haines S. Fibrinolytic therapy for intraventricular hemorrhage in adults. Cochrane Database Syst Rev 2002;(03): CD003692

53 Gaberel T, Magheru C, Parienti JJ, Huttner HB, Vivien D, Emery E. Intraventricular fibrinolysis versus external ventricular drainage alone in intraventricular hemorrhage: a meta-analysis. Stroke 2011;42(10):2776-2781

54 Naff N, Williams MA, Keyl PM, et al. Low-dose recombinant tissue-type plasminogen activator enhances clot resolution in brain hemorrhage: the intraventricular hemorrhage thrombolysis trial. Stroke 2011;42(11):3009-3016

55 Khan NR, Tsivgoulis G, Lee SL, et al. Fibrinolysis for intraventricular hemorrhage: an updated meta-analysis and systematic review of the literature. Stroke 2014;45(09):2662-2669

56 Moradiya Y, Murthy SB, Newman-Toker DE, Hanley DF, Ziai WC. Intraventricular thrombolysis in intracerebral hemorrhage requiring ventriculostomy: a decade-long real-world experience. Stroke 2014;45(09):2629-2635

57 Varelas PN, Rickert KL, Cusick J, et al. Intraventricular hemorrhage after aneurysmal subarachnoid hemorrhage: pilot study of treatment with intraventricular tissue plasminogen activator. Neurosurgery 2005;56(02):205-213, discussion 205-213

58 Hanley DF, Lane K, McBee N, et al; CLEAR III Investigators. Thrombolytic removal of intraventricular haemorrhage in treatment of severe stroke: results of the randomised, multicentre, multiregion, placebo-controlled CLEAR III trial. Lancet 2017;389 (10069):603-611

59 Litrico S, Almairac F, Gaberel T, et al. Intraventricular fibrinolysis for severe aneurysmal intraventricular hemorrhage: a randomized controlled trial and meta-analysis. Neurosurg Rev 2013; 36(04):523-530, discussion 530-531

60 Ramakrishna R, Sekhar LN, Ramanathan D, et al. Intraventricular tissue plasminogen activator for the prevention of vasospasm and hydrocephalus after aneurysmal subarachnoid hemorrhage. Neurosurgery 2010;67(01):110-117, discussion 117

61 Findlay JM, Jacka MJ. Cohort study of intraventricular thrombolysis with recombinant tissue plasminogen activator for aneurysmal intraventricular hemorrhage. Neurosurgery 2004;55(03): 532-537, discussion 537-538

62 Ziai WC, Tuhrim S, Lane K, et al; CLEAR III Investigators. A multicenter, randomized, double-blinded, placebo-controlled phase III study of Clot Lysis Evaluation of Accelerated Resolution of Intraventricular Hemorrhage (CLEAR III). Int J Stroke 2014;9 (04):536-542
63 Rabinstein AA. Intracerebral haemorrhage: no good treatment but treatment helps. Lancet 2017;389(10069):575-576

64 Staykov D, Huttner HB, Lunkenheimer J, et al. Single versus bilateral external ventricular drainage for intraventricular fibrinolysis in severe ventricular haemorrhage. J Neurol Neurosurg Psychiatry 2010;81(01):105-108

65 Hinson HE, Melnychuk E, Muschelli J, Hanley DF, Awad IA, Ziai WC. Drainage efficiency with dual versus single catheters in severe intraventricular hemorrhage. Neurocrit Care 2012;16 (03):399-405

66 Du B, Wang J, Zhong XL, et al. Single versus bilateral external ventricular drainage for intraventricular fibrinolysis using urokinase in severe ventricular haemorrhage. Brain Inj 2014;28(11): 1413-1416

67 Ziai W, Awad I, Hanley D. Efficiency of intraventricular hemorrhage removal in the clot lysis: evaluation of Accelerated Resolution of Intraventricular Hemorrhage Trial (CLEAR III). In: Abstract Number: AS02-020. 3rd European Stroke Organization Conference (ESOC 2017), Prague, Czech; 2017

68 Naff NJ, Williams MA, Rigamonti D, Keyl PM, Hanley DF. Blood clot resolution in human cerebrospinal fluid: evidence of firstorder kinetics. Neurosurgery 2001;49(03):614-619, discussion 619-621

69 Hanley D. Final results CLEARIVH Trial: clot lysis, safety and 30 day functional outcomes. In: Abstract Presentation at the European Stroke Conference, Nice, France, May 13-16, 2008

70 Wang YC, Lin CW, Shen CC, Lai SC, Kuo JS. Tissue plasminogen activator for the treatment of intraventricular hematoma: the dose-effect relationship. J Neurol Sci 2002;202(1-2):35-41

71 Ducruet AF, Hickman ZL, Zacharia BE, et al. Exacerbation of perihematomal edema and sterile meningitis with intraventricular administration of tissue plasminogen activator in patients with intracerebral hemorrhage. Neurosurgery 2010;66(04): 648-655

72 Ziai W, Moullaali T, Nekoovaght-Tak S, et al. No exacerbation of perihematomal edema with intraventricular tissue plasminogen activator in patients with spontaneous intraventricular hemorrhage. Neurocrit Care 2013;18(03):354-361

73 Volbers B, Wagner I, Willfarth W, Doerfler A, Schwab S, Staykov D. Intraventricular fibrinolysis does not increase perihemorrhagic edema after intracerebral hemorrhage. Stroke 2013;44(02): 362-366

74 Kornbluth J, Nekoovaght-Tak S, Ullman N, Carhuapoma JR, Hanley DF, Ziai W. Early quantification of hematoma Hounsfield units on noncontrast CT in acute intraventricular hemorrhage predicts ventricular clearance after intraventricular thrombolysis. AJNR Am J Neuroradiol 2015;36(09):1609-1615

75 Ziai WC, Muschelli J, Thompson CB, et al. Factors affecting clot lysis rates in patients with spontaneous intraventricular hemorrhage. Stroke 2012;43(05):1234-1239

76 Broderick J, Lu M, Jackson C, et al; NINDS t-PA Stroke Study Group. Apolipoprotein E phenotype and the efficacy of intravenous tissue plasminogen activator in acute ischemic stroke. Ann Neurol 2001;49(06):736-744

77 González-Conejero R, Fernández-Cadenas I, Iniesta JA, et al; Proyecto Ictus Research Group. Role of fibrinogen levels and factor XIII V34L polymorphism in thrombolytic therapy in stroke patients. Stroke 2006;37(09):2288-2293

78 Fernandez-Cadenas I, Alvarez-Sabin J, Ribo M, et al. Influence of thrombin-activatable fibrinolysis inhibitor and plasminogen activator inhibitor-1 gene polymorphisms on tissue-type plasminogen activator-induced recanalization in ischemic stroke patients. J Thromb Haemost 2007;5(09):1862-1868

79 Fernández-Cadenas I, Del Río-Espínola A, Giralt D, et al. IL1B and VWF variants are associated with fibrinolytic early recanalization in patients with ischemic stroke. Stroke 2012;43(10): 2659-2665 
80 Montaner J, Fernández-Cadenas I, Molina CA, et al. Safety profile of tissue plasminogen activator treatment among stroke patients carrying a common polymorphism (C-1562T) in the promoter region of the matrix metalloproteinase- 9 gene. Stroke 2003;34(12):2851-2855

81 Staykov D, Kuramatsu JB, Bardutzky J, et al. Efficacy and safety of combined intraventricular fibrinolysis with lumbar drainage for prevention of permanent shunt dependency after intracerebral hemorrhage with severe ventricular involvement: A randomized trial and individual patient data meta-analysis. Ann Neurol 2017;81(01):93-103

82 Wolf S. EARLYdrain - Outcome after Early Lumbar CSF-drainage in Aneurysmal SAH (EARLYDRAIN). Identifier: NCT01258257. Available at: https://clinicaltrials.gov/ct2/show/NCT01258257. Accessed 2016

83 Diringer MN, Edwards DF, Zazulia AR. Hydrocephalus: a previously unrecognized predictor of poor outcome from supratentorial intracerebral hemorrhage. Stroke 1998;29(07):1352-1357

84 Adams RE, Diringer MN. Response to external ventricular drainage in spontaneous intracerebral hemorrhage with hydrocephalus. Neurology 1998;50(02):519-523

85 Phan TG, Koh M, Vierkant RA, Wijdicks EF. Hydrocephalus is a determinant of early mortality in putaminal hemorrhage. Stroke 2000;31(09):2157-2162

86 Liliang PC, Liang CL, Lu CH, et al. Hypertensive caudate hemorrhage prognostic predictor, outcome, and role of external ventricular drainage. Stroke 2001;32(05):1195-1200

87 Bhattathiri PS, Gregson B, Prasad KS, Mendelow AD; STICH Investigators. Intraventricular hemorrhage and hydrocephalus after spontaneous intracerebral hemorrhage: results from the STICH trial. Acta Neurochir Suppl (Wien) 2006;96:65-68

88 Woo D, Kruger AJ, Sekar P, et al. Incontinence and gait disturbance after intraventricular extension of intracerebral hemorrhage. Neurology 2016;86(10):905-911

89 Coplin WM, Vinas FC, Agris JM, et al. A cohort study of the safety and feasibility of intraventricular urokinase for nonaneurysmal spontaneous intraventricular hemorrhage. Stroke 1998;29(08): 1573-1579

90 Ziai WC, Torbey MT, Naff NJ, et al. Frequency of sustained intracranial pressure elevation during treatment of severe intraventricular hemorrhage. Cerebrovasc Dis 2009;27(04):403-410

91 Ziai WC, Melnychuk E, Thompson CB, Awad I, Lane K, Hanley DF. Occurrence and impact of intracranial pressure elevation during treatment of severe intraventricular hemorrhage. Crit Care Med 2012;40(05):1601-1608

92 Hinson HE, Hanley DF, Ziai WC. Management of intraventricular hemorrhage. Curr Neurol Neurosci Rep 2010;10(02):73-82
93 Nyquist P, Hanley DF. The use of intraventricular thrombolytics in intraventricular hemorrhage. J Neurol Sci 2007;261(12):84-88

94 Chen Q Tang J, Tan L, et al. Intracerebral hematoma contributes to hydrocephalus after intraventricular hemorrhage via aggravating iron accumulation. Stroke 2015;46(10):2902-2908

95 Fam MD, Pang A, Zeineddine HA, et al; CLEAR III Trial Investigators. Demographic risk factors for vascular lesions as etiology of intraventricular hemorrhage in prospectively screened cases. Cerebrovasc Dis 2017;43(5-6):223-230

96 Chen CC, Liu CL, Tung YN, et al. Endoscopic surgery for intraventricular hemorrhage (IVH) caused by thalamic hemorrhage: comparisons of endoscopic surgery and external ventricular drainage (EVD) surgery. World Neurosurg 2011;75(02):264-268

97 Zhang Z, Li X, Liu Y, Shao Y, Xu S, Yang Y. Application of neuroendoscopy in the treatment of intraventricular hemorrhage. Cerebrovasc Dis 2007;24(01):91-96

98 Li Y, Zhang H, Wang X, et al. Neuroendoscopic surgery versus external ventricular drainage alone or with intraventricular fibrinolysis for intraventricular hemorrhage secondary to spontaneous supratentorial hemorrhage: a systematic review and meta-analysis. PLoS One 2013;8(11):e80599

99 Naff NJ, Hanley DF, Keyl PM, et al. Intraventricular thrombolysis speeds blood clot resolution: results of a pilot, prospective, randomized, double-blind, controlled trial. Neurosurgery 2004;54(03):577-583, discussion 583-584

100 Naff NJ, Carhuapoma JR, Williams MA, et al. Treatment of intraventricular hemorrhage with urokinase: effects on 30-Day survival. Stroke 2000;31(04):841-847

101 Tung MY, Ong PL, Seow WT, Tan KK. A study on the efficacy of intraventricular urokinase in the treatment of intraventricular haemorrhage. Br J Neurosurg 1998;12(03):234-239

102 Akdemir H, Selçuklu A, Paşaoğlu A, Oktem IS, Kavuncu I. Treatment of severe intraventricular hemorrhage by intraventricular infusion of urokinase. Neurosurg Rev 1995;18(02):95-100

103 Torres A, Plans G, Martino J, et al. Fibrinolytic therapy in spontaneous intraventricular haemorrhage: efficacy and safety of the treatment. Br J Neurosurg 2008;22(02):269-274

104 Huttner HB, Tognoni E, Bardutzky J, et al. Influence of intraventricular fibrinolytic therapy with rt-PA on the long-term outcome of treated patients with spontaneous basal ganglia hemorrhage: a case-control study. Eur J Neurol 2008;15(04):342-349

105 Rainov NG, Burkert WL. Urokinase infusion for severe intraventricular haemorrhage. Acta Neurochir (Wien) 1995;134(1-2):55-59

106 Todo T, Usui M, Takakura K. Treatment of severe intraventricular hemorrhage by intraventricular infusion of urokinase. J Neurosurg 1991;74(01):81-86 